\title{
Inhibitory effects of fermented brown rice and rice bran on the development of acute hepatitis in Long-Evans Cinnamon rats
}

\author{
TOSHIYUKI SHIBATA ${ }^{1}$, HIROKI NAGAYASU ${ }^{2}$, HIROYUKI KITAJO² ${ }^{2}$ MAKOTO ARISUE ${ }^{2}$, \\ TOMOMI YAMASHITA ${ }^{1}$, DAIJIRO HATAKEYAMA ${ }^{1}$, TERUAKI IWASAKI ${ }^{3}$ and HIROSHI KOBAYASHI ${ }^{4}$ \\ ${ }^{1}$ Department of Oral and Maxillofacial Sciences, Gifu University Graduated School of Medicine, 1-1 Yanagido, \\ Gifu 501-1194; ${ }^{2}$ Second Department of Oral and Maxillofacial Surgery, Faculty of Dentistry, Health Sciences, \\ University of Hokkaido, Tobetsu, Hokkaido 061-0293; ${ }^{3}$ Genmai Koso Co., Ltd., Nishi-1, Kita-12, Kita-ku, \\ Sapporo 001-0012; ${ }^{4}$ Sapporo Cancer Seminar, 6 Osori-Nishi, Chuo-ku, Sapporo 064-0820, Japan
}

Received June 21, 2005; Accepted August 22, 2005

\begin{abstract}
To investigate the effects of fermented brown rice (FBRA) on the development of hereditary hepatitis in LongEvans Cinnamon (LEC) rats, we compared the incidence and grades of acute hepatitis among rats fed 5\% and 10\% FBRA in the conventional diet and the conventional diet alone. Both the 5\% and 10\% FBRA-supplemented diets indicated a tendency to prevent the development of hepatitis, and the significant effect of 10\% FBRA was observed until 16-17 weeks of age in the accumulated incidence and survival ratio compared with the unsupplemented conventional diet, although no significant difference was observed between $5 \%$ and $10 \%$ FBRA-supplemented diets. At the age of 12 weeks, which is just before the rats develop hepatitis, serum copper levels in rats fed either of the test diets were similar to those in rats fed the conventional diet. Furthermore, the copper concentration in liver tissue at 12 weeks of age was not changed by the test diet. These results suggest that FBRA has preventive effects on the development of hepatitis in LEC rats and may play an important role in protecting the liver against the free radicals induced by copper accumulation in the liver.
\end{abstract}

\section{Introduction}

The LEC rat (Long-Evans rat with a cinnamon-like coat) is an inbred strain of a mutant rat isolated from a closed colony of Long-Evans rats. LEC rats spontaneously develop acute

Correspondence to: Dr Toshiyuki Shibata, Department of Oral and Maxillofacial Sciences, Gifu University, Graduated School of Medicine, 1-1 Yanagido, Gifu 501-1194, Japan

E-mail: shibat@cc.gifu-u.ac.jp

Abbreviations: LEC rat, Long-Evans Cinnamon rat; FBRA, fermented brown rice

Key words: Long-Evans Cinnamon rat, hepatitis, fermented brown rice, rice bran hepatitis accompanied with severe jaundice about 3-4 months after birth, and most of them die of fulminant hepatitis. Those that survive acute hepatitis suffer from chronic hepatitis for over a year, and nearly $100 \%$ of them ultimately develop cholangiofibrosis and hepatocellular carcinoma (1). LEC rats are thus regarded as a useful animal model for research on human hepatic diseases including hepatocarcinogenesis. In the LEC rat liver, excessive amounts of copper accumulate due to the mutation of the Atp $7 \mathrm{~b}$ gene, a rat homologue to the human Wilson's disease gene, which encodes a coppertransporting P-type ATPase (2). Copper accumulation was proved to be genetically linked with hepatitis development, and copper accumulation in the liver was revealed to start at 2 days of age and gradually increase to hepatotoxic levels until the onset of hepatitis (3-5). It has also been proposed that the excess copper catalyzes the formation of highly toxic hydroxyl radicals by way of a Fenton-like reaction, and selenium-dependent glutathione peroxidase (GSH-Px) is reduced by $40 \%$ in the LEC rat liver (6). The level of 8-hydroxyguanosine, an oxidative DNA damaging product, is elevated in LEC rat livers at the age of 3-4 months when clinical symptoms of acute hepatitis appear (7). Thus, the impaired antioxidant defense is speculated to play an important role in the development of hepatitis and hepatocellular carcinoma in LEC rats.

On the other hand, it has been recognized that dietary factors play an important role in the prevention of several types of cancer. Rice, one of the major cereals, is consumed as a staple food among worldwide populations, especially Asian countries, and contains several kinds of antioxidants, such as ferulic acid (8), phytic acid (9), tocophenols and oryzanols (10), especially in the bran or germ. Rice germ itself or the compounds of the rice bran or rice germ have chemopreventive effects against carcinogenesis in the large intestine or tongue of rats (11-14). Brown rice fermented by Aspergillus Oryzae (FBRA) was used in this study, which is a processed food prepared by fermenting brown rice and rice bran with Aspergillus Oryzae. It is already known that FBRA acts as a potent free radical scavenger (15). It has also been revealed that FBRA has inhibitory effects on 
Table I. Composition of FBRA.

Amount/100 g FBRA

\begin{tabular}{lr}
\hline Hydrosis & $3.3 \mathrm{~g}$ \\
Protein & $23.8 \mathrm{~g}$ \\
Fat & $20.5 \mathrm{~g}$ \\
Ash & $9.0 \mathrm{~g}$ \\
Hydrocarbon & $22.4 \mathrm{~g}$ \\
Fiber & $21.0 \mathrm{~g}$ \\
Phytic acid & $3.86 \mathrm{~g}$ \\
Vitamin A & $0.003 \mathrm{mg}$ \\
Vitamin B group & $4.97 \mathrm{mg}$ \\
Vitamin E group & $15.0 \mathrm{mg}$ \\
Vitamin K group & $63.0 \mathrm{mg}$ \\
Sodium & $8.1 \mathrm{mg}$ \\
Phosphorus & $1.96 \mathrm{~g}$ \\
Calcium & $308.0 \mathrm{mg}$ \\
Iron & $9.9 \mathrm{mg}$ \\
Magnesium & $810.0 \mathrm{mg}$ \\
Copper & $936.0 \mathrm{mg}$ \\
Zinc & $5.99 \mathrm{mg}$ \\
Manganese & $15.6 \mathrm{mg}$ \\
Selenium & $8.0 \mu \mathrm{g}$ \\
Arabionoxylane & $5.6 \mathrm{~g}$ \\
\hline &
\end{tabular}

chemically induced carcinogenesis in the colon (16), esophagus (17) and liver (18) in well-established preclinical models. In the present study, we examined the effects of FBRA on the development of acute hepatitis with an aim at preventing hepatocellular carcinoma in LEC rats.

\section{Materials and methods}

Animals and diets. Female LEC rats (Charles River Animal Center, Tokyo, Japan) obtained at 4 weeks of age were housed in wire cages ( 3 or 4 rats/cage) under controlled conditions of humidity (50-60\%), lighting (12 h light/dark cycle) and temperature $\left(23 \pm 2^{\circ} \mathrm{C}\right)$, with free access to water and a basal CE-2 diet (Nippon Clea Co., Tokyo, Japan) at the Center for Experimental Animals, Health Sciences University of Hokkaido. The CE-2 diet was used as the basal diet throughout the study. Test diets were prepared by supplementing the conventional diet (CE-2) with FBRA. At 8 weeks of age, the rats were divided randomly into three groups and given the conventional diet (CE-2) or test diets containing $5 \%$ or $10 \%$ weight of FBRA and the same experiment was performed in triplicate to confirm the results as the incidence and grades of acute hepatitis varied depending on the birth groups. This animal study was approved by the Committee of Health Sciences at University of Hokkaido on the treatment of experimental animals.

Chemicals. FBRA was supplied by Genmai Koso Co., Ltd. (Sapporo, Japan). Briefly, for the processing of FBRA, a

Table II. Effects of the diets on the incidence of hepatitis in LEC rats.

\begin{tabular}{lcccr}
\hline & \multicolumn{4}{c}{ Incidence of hepatitis/no. of rats $(\%)^{\mathrm{b}}$} \\
\cline { 2 - 5 } Dietary group $^{\mathrm{a}}$ & Experiment 1 & Experiment 2 & Experiment 3 & Total \\
\hline Conventional & $10 / 10(100)$ & $10 / 10(100)$ & $27 / 29(93)$ & $47 / 49(96)$ \\
$5 \%$ FBRA & $9 / 10(90)$ & $9 / 9(100)$ & $22 / 26(85)$ & $40 / 45(89)$ \\
$10 \%$ FBRA & $6 / 9(67)$ & $10 / 10(100)$ & $25 / 29(86)$ & $41 / 48(85)$ \\
\hline
\end{tabular}

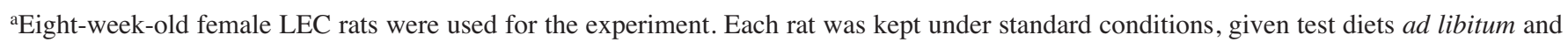

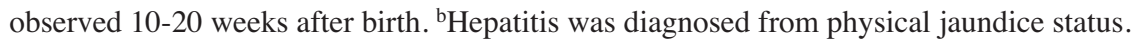

Table III. Changes in the age of LEC rats fed with the test diets at the development of hepatitis.

\begin{tabular}{lccr}
\hline & \multicolumn{3}{c}{ Age at the onset of hepatitis (no. ${ }^{\mathrm{b}}$ of rats observed) } \\
\cline { 2 - 4 } Dietary group $^{\mathrm{a}}$ & Experiment 1 & Experiment 2 & Experiment 3 \\
\hline Conventional & $101.9 \pm 4.2$ days $(10)$ & $110.2 \pm 2.6$ days $(10)$ & $110.4 \pm 4.4$ days $(27)$ \\
5\%FBRA & $102.9 \pm 4.1$ days $(10)$ & $111.2 \pm 2.9$ days $(9)$ & $112.2 \pm 3.9$ days $(22)$ \\
$10 \%$ FBRA & $104.9 \pm 4.7$ days $(9)$ & $111.0 \pm 2.7$ days (10) & $112.1 \pm 4.7$ days $(25)$ \\
\hline
\end{tabular}

${ }^{a}$ Eight-week-old female LEC rats were used for the experiment. Each rat was kept under standard conditions, given test diets ad libitum and observed 10-20 weeks after birth. ${ }^{b}$ Development of hepatitis was diagnosed from physical jaundice status. Data are means \pm SE of rats that developed hepatitis in each experiment. 


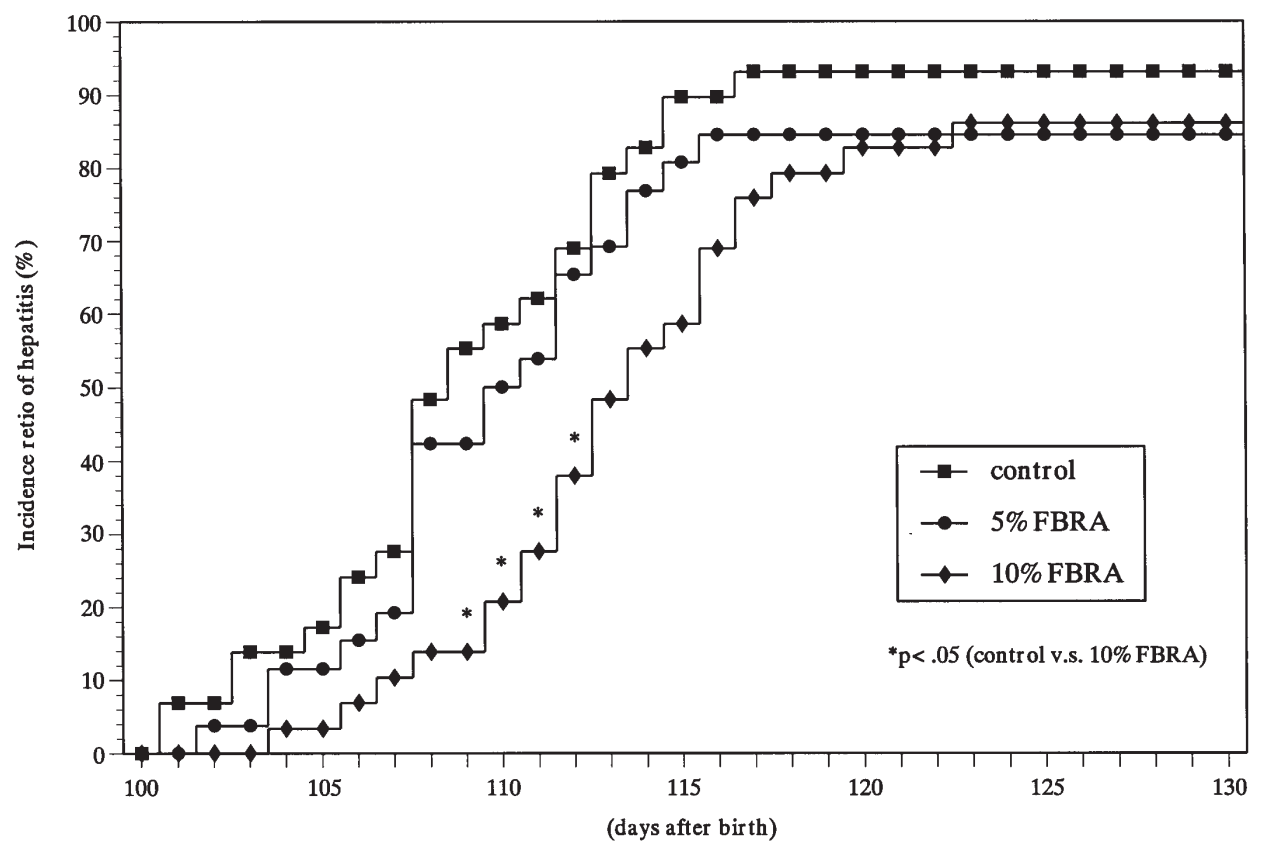

Figure 1. Accumulated incidence ratio of hepatitis in experiment 3 . The accumulated hepatitis incidence ratio at 109 to 112 days of age was significantly reduced by the test diet supplemented with 10\% FBRA, although no significant difference was observed between the 5\% and $10 \%$ FBRA-supplemented diets.

fermentation base is made by steaming brown rice and rice bran. Aspergillus Oryzae is then seeded in the fermentation base, and fermentation is continued for 18-24 h. Subsequently, a second fermentation is continued for an additional 12-24 h for aging. The fermented product is then dried and powdered. The final composition of FBRA is shown in Table I.

Blood collection and analysis of serum copper levels. Blood samples were collected from the jugular vein of every rat on respective days, and serum copper concentrations of these blood samples were determined with a Zeeman polarized atomic absorption spectrophotometer (model Z-8100; Hitachi, Tokyo, Japan).

Examination of copper concentration in the liver tissue. Female LEC rats aged 12 weeks that had not yet developed acute hepatitis were used for the analysis of copper concentrations in the liver. The liver was removed, weighed, and stored at $-80^{\circ} \mathrm{C}$. The tissues were wet ashed with nitric, perchloric, and sulfuric acids, and copper concentrations were determined with an atomic absorption spectrophotometer (model 180-30; Hitachi Ltd., Tokyo, Japan).

Detection and diagnosis of hepatitis. The LEC rats were observed daily for jaundice, and were diagnosed as having hepatitis upon the appearance of icteric ears and foot pads in accordance with previous reports (1).

Statistical analysis. Statistically significant differences between the groups were determined using the $\chi^{2}$ test or Student's t-test. In all cases, p-values $<0.05$ were considered significant.

\section{Results}

Effects of test diets on the incidence of acute hepatitis in LEC rats. Acute hepatitis was observed in 96\% (47/49) of rats fed a conventional diet, and $89 \%(41 / 48)$ and $85 \%$ (14/48) of rats fed the $5 \%$ and $10 \%$ FBRA-supplemented diets, respectively. Thus, both the 5\% and 10\% FBRA test diets indicated an inhibitory action compared with the conventional diet, although the difference was not significant in any of the experiments or average result (Table II).

Effects of test diets on the duration of hepatitis-free periods. Hepatitis-free periods were prolonged in the rats fed test diets of both $5 \%$ and $10 \%$ FBRA compared with that in rats fed the conventional diet in every experiment (Table III), although a significant difference was not observed. To evaluate the effects of test diets in detail, we examined the accumulated hepatitis incidence ratio in experiment 3. During 109 to 112 days of age, the ratio was significantly reduced by the $10 \%$ FBRA-supplemented test diet, although a significant difference was not observed between the 5\% and 10\% FBRA-supplemented diets (Fig. 1).

Effects of test diets on the mortality and survival time. In the serial experiments, all LEC rats died of fulminant hepatitis 3 or 4 days after the onset of acute hepatitis. The average survival period was not significantly prolonged in rats fed either test diet with $5 \%$ or $10 \%$ FBRA, compared with that in rats fed the conventional diet, although a significant difference was observed at 114 to 118 days of age in the accumulated survival rate (Table IV) (Fig. 2).

These results indicated that the diets supplemented with FBRA possibly prevented the onset of hepatitis and/or significantly prolonged the hepatitis-free period before the onset of acute hepatitis, which is a hypercarcinogenic state of LEC rats.

Effects on the serum copper levels and liver tissue copper concentrations. To evaluate the effect of copper accumulation in LEC rats, 10 rats were used in each group. Serum samples 
Table IV. Changes in the survival period of LEC rats fed the test diets.

Age (days) at death (no. of rats observed)

\begin{tabular}{llll}
\hline Dietary group $^{\mathrm{a}}$ & Experiment 1 & Experiment 2 & Experiment 3 \\
\hline Conventional & $104.3 \pm 5.1$ days $(10)$ & $113.1 \pm 2.1$ days $(10)$ & $112.7 \pm 4.3$ days $(27)$ \\
$5 \%$ FBRA & $103.5 \pm 4.7$ days $(10)$ & $113.8 \pm 2.2$ days $(9)$ & $114.9 \pm 4.3$ days $(22)^{\mathrm{b}}$ \\
$10 \%$ FBRA & $106.6 \pm 3.6$ days (9) & $114.4 \pm 3.1$ days (10) & $115.2 \pm 4.7$ days $(25)^{\mathrm{c}}$
\end{tabular}

${ }^{a}$ Eight-week-old female LEC rats were used for the experiment. Each rat was kept under standard conditions, given test diets ad libitum and observed 10-20 weeks after birth. Data are means \pm SE of rats that developed hepatitis in each experiment. No significant difference was observed $\left({ }^{\mathrm{b}} \mathrm{p}=0.0846\right.$ and $\left.{ }^{\mathrm{c}} \mathrm{p}=0.0589\right)$.

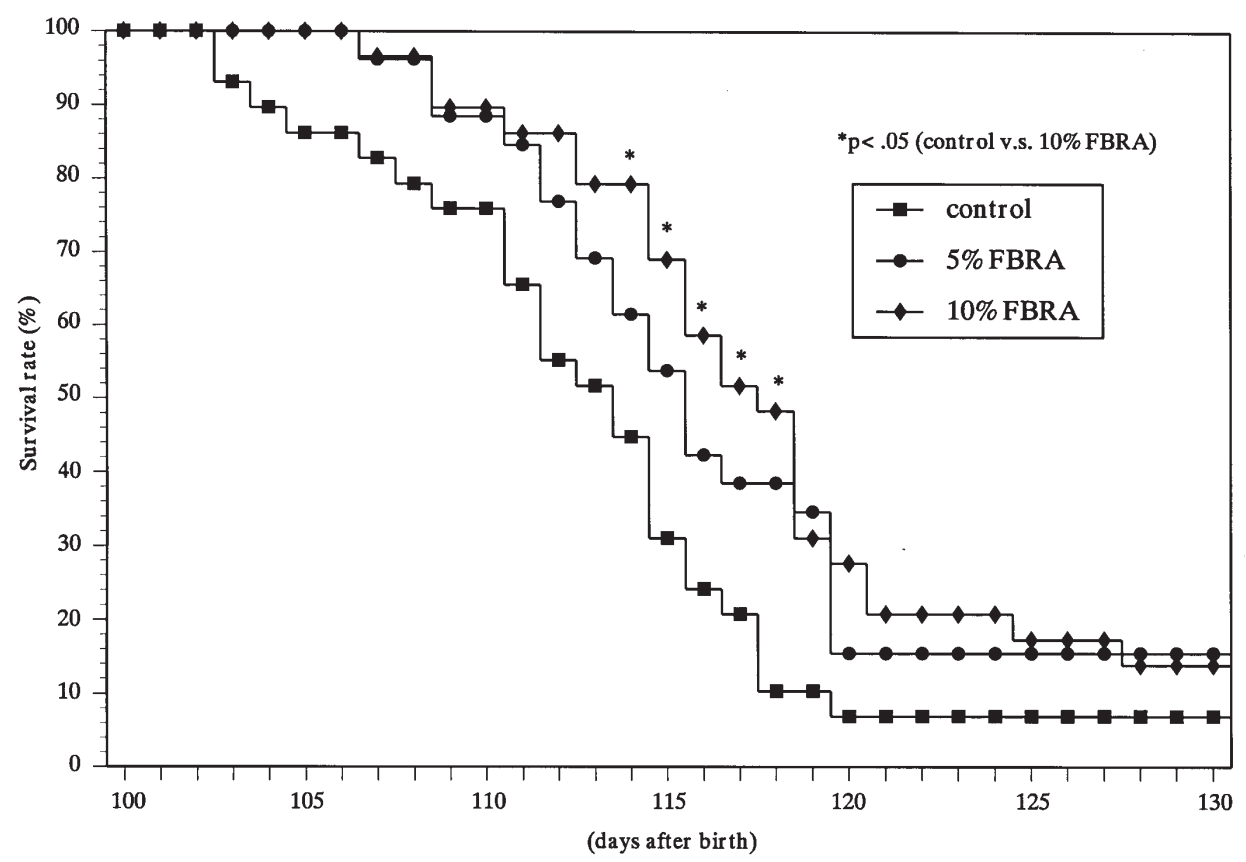

Figure 2. Accumulated survival ratio in experiment 3. A significant difference was observed at 114 to 118 days after birth in the accumulated survival rate.

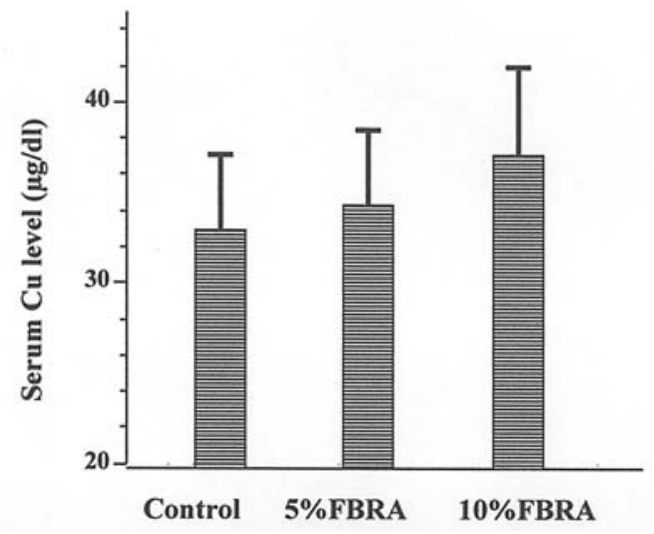

Figure 3. Effect of test diets on the serum copper levels. Serum samples were obtained from LEC rats at 12 weeks of age. These rats were used for the analysis of copper concentration in the liver tissue following serum sampling. No significant difference was observed among the groups.

obtained from LEC rats aged 12 weeks indicated the same levels of copper concentration among the groups (Fig. 3). These rats were then sacrificed, and the liver tissues were removed for the analysis of copper concentration following
Table V. Effects of the test diets on the copper concentration in the liver tissue.

\begin{tabular}{lc}
\hline Dietary group $^{\mathrm{a}}$ & $\begin{array}{c}\text { Copper concentration } \\
\mu \mathrm{g} / \mathrm{g} \text { wet weight }\end{array}$ \\
\hline Conventional $(\mathrm{n}=10)$ & $426.1 \pm 125.1$ \\
5\%FBRA $(\mathrm{n}=10)$ & $509.9 \pm 77.5$ \\
$10 \%$ FBRA $(\mathrm{n}=10)$ & $472.5 \pm 175.6$ \\
\hline
\end{tabular}

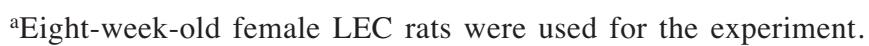
Each rat was kept under standard conditions and given test diets

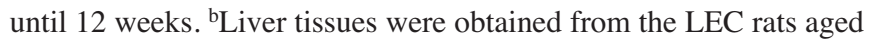
12 weeks that had not yet developed acute hepatitis. Copper concentration was determined by atomic absorption spectrophotometry. Data are means \pm SE of 10 rats in each group.

serum sampling. The liver tissues in each group also contained the same amounts of copper (Table V). To evaluate the liver condition, serum transaminases (AST and ALT) were measured. Both AST and ALT levels were within normal 
range, and these findings corresponded well to the physical status (data not shown).

\section{Discussion}

The results of the present study show that FBRA possibly prevents the development of hepatitis, which is a hypercarcinogenic state of LEC rats, and prolongs the hepatitisfree period and survival time, but does not affect the accumulation of copper in liver tissues.

LEC rats are known to have hereditary abnormal copper metabolism by which copper accumulates in the liver, brain, kidney and other organs shortly after birth, and this accumulation of copper in excess of metabolic requirements causes fatal hepatocellular injury. Indeed, the oral administration of D-penicillamine, a copper-chelating agent widely used in the treatment of Wilson's disease, completely inhibits the development of hepatitis and hepatocellular carcinoma $(19,20)$. Furthermore, the administration of fatty acids can strongly stimulate bile juice secretion from the liver and may consequently promote excretion of copper, leading to the prevention of hepatitis in LEC rats (21). The accumulated copper appears to interact with physiologically produced active oxygen species, such as superoxide anion and hydrogen peroxide, catalyzing the formation of highly toxic hydroxyl radicals by way of a Fenton-like reaction $(22,23)$. Moreover, it is reported that the antioxidant defense, such as glutathione peroxidase, is reduced by $40 \%$ in LEC rat livers (6). Thus, an accumulation of copper and radicals play an important role in the development hepatitis and hepatocellular carcinoma in LEC rats. This is the first study to report that hepatitis of LEC rats is prevented without affecting the copper accumulation.

FBRA is a processed food prepared by fermenting brown rice and rice bran with Aspergillus Oryzae. The advantage of fermentation has been recognized in recent years, but the details are not well known. Japanese traditional fermented soybean products, such as soybean paste (miso) or soy sauce (shoyu), which are prepared by fermenting soybeans with Aspergillus Oryzae, are more stable against lipid peroxidation than unfermented soybean. Esaki et al reported that soybeans produce antioxidants, 6-hydroxydaidzein, 8-dehydroxydaidzein and 8-hydroxygenistein by fermenting with Aspergillus Oryzae (24). These agents are reported to possess high DPPH radical-scavenging and antiproliferative activity (25). In epidemiological or preclinical studies, some evidence on the preventive effects of such fermented soybean products has been reported (26-28). However, little is understood about the efficiency of fermented rice. It is possible that the fermentation of rice with Aspergillus Oryzae has similar functional aspects to the fermentation of soybeans. It is also possible that the efficiency of FBRA is mediated through the action of several trace elements including selenium, which is biotransformed into organic form during the fermentation of brown rice and rice bran (29-31). Furthermore, the antioxidant activity of FBRA is also suggested to be significantly associated with the suppression of hepatocarcinogenesis in previous studies using well-established chemical carcinogenesis models (16-18).

In this study, FBRA did not change the level of copper accumulation in liver tissues, but prevented hepatitis. No report has shown suppression of hepatitis development in LEC rats without reducing the copper accumulation in the liver tissues until now. This strongly suggests that FBRA may moderate the events following copper accumulation, including the generation of free radicals and/or augmentation of radical scavenging ability.

In conclusion, the present study proved the chemopreventive effect of FBRA on the hepatitis in LEC rats that renders them prone to carcinogesis. While the mechanism of FBRA is yet to be elucidated, our data imply that dietary FBRA can potentially assist in reducing human liver diseases progressed by free radicals. To clarify this point, further examination is necessary.

\section{Acknowledgements}

This work was supported in part by a Grant-in-Aid for Scientific Research from the Japanese Ministry of Education, Science, Sports and Culture and from Promotion and Mutual Aid Corporation for Private Schools of Japan. We thank Ms. Masako Yanome for her assistance in preparing the manuscript.

\section{References}

1. Takeichi N, Kobayashi H, Yoshida MC, Sasaki M, Dempo K and Mori M: Spontaneous hepatitis in Long-Evans rats, a potential animal model for fluminant hepatitis in man. Acta Pathol Jpn 38: 1369-1375, 1988.

2. Wu J, Forbes JR, Chen HS and Cox DW: The LEC rat has a deletion in the copper transporting ATPase gene homologous to the Wilson disease gene. Nat Genet 7: 541-545, 1994.

3. Sasaki N, Hayashizaki Y, Muramatsu M, Matsuda Y, Ando Y, Kuramoto T, Serikawa T, Azuma T, Naito A, Agui T, Yamashita T, Miyoshi I, Takeichi N and Kasai N: The gene responsible for LEC hepatitis, located on rat chromosome 16, is the homologue to the human Wilson disease gene. Biochem Biophys Res Commun 202: 512-518, 1994.

4. Li Y, Togashi Y, Sato S, Enomoto T, Kang J-H, Takeichi N, Kobayashi H, Kojima Y, Une Y and Uchino J: Abnormal copper accumulation in non-cancerous and cancerous liver tissues of LEC rats developing hepatitis and spontaneous hepatoma. Jpn J Cancer Res 82: 490-492,1991.

5. Li Y, Togashi Y, Sato S, Enomoto T, Kang J-H, Takeichi N, Kobayashi H, Kojima Y, Une Y and Uchino J: Spontaneous hepatic copper accumulation in Long-Evans Cinnamon rats with hereditary hepatitis. J Clin Invest 87: 1858-1861, 1991.

6. Suemizu H, Yoshimura S, Takeichi N and Moriuchi T: Decreased expression of liver glutathione peroxidase in LongEvans Cinnamon mutant rats predisposed to hepatitis and hepatoma. Hepatology 19: 694-700, 1994.

7. Yamamoto F, Kasai H, Togashi Y, Takeichi N, Hori T and Nishimura S: Elevated level of 8-hydroxydeoxyguanosine in DNA of liver, kidney, and brain of Long-Evans Cinnamon rats. Jpn J Cancer Res 84: 508-511, 1993.

8. Taniguchi H, Hosoda A, Tsuno T, Maruta Y and Nomura E: Preparation of ferulic acid and its application for the synthesis of cancer preventive agents. Anticancer Res 19: 3757-3761, 1999.

9. Graf E, Empson KL and Eaton JW: Phytic acid. A natural antioxidant. J Biol Chem 262: 11647-11650, 1987.

10. Jariwalla RJ: Rice-bran products; phytonutrients with potential applications in preventive and clinical medicine. Drugs Exp Clin Res 27: 17-26, 2001.

11. Kawabata K, Yamamoto T Hara A, Shimizu M, Yamada Y, Matsunaga K, Tanaka T and Mori H: Modifying effects of ferulic acid on azoxymethane-induced colon carcinogenesis in F344 rats. Cancer Lett 157: 15-21, 2000.

12. Kawabata K, Tanaka T, Murakami T, Okada T, Murai H, Yamamoto T, Hara A, Shimizu M, Yamada Y, Matsunaga K, Kuno T, Yoshimi N, Sugie S and Mori H: Dietary prevention of azoxymethane-induced colon carcinogenesis with rice-germ in F344 rats. Carcinogenesis 20: 2109-2115, 1999. 
13. Mori H, Kawabata K, Yoshimi N, Tanaka T, Murakami T, Okada T and Murai H: Chemopreventive effects of ferulic acid on oral and rice germ on large bowel carcinogesis. Anticancer Res 19: 3775-3778, 1999.

14. Tanaka T, Kojima T, Kawamori T, Wang A, Suzuki M, Okamoto $\mathrm{K}$ and Mori H: Inhibition of 4-nitroquinoline-1-oxideinduced rat tongue carcinogenesis by the naturally occurring plant phenolics caffelic, ellagic, chlorogenic and ferulic acids. Carcinogenesis 14: 1321-1325, 1993.

15. Tazawa K, Naoko F and Hirohide N: Superoxide scavenging effect of fermented brown rice determined by ESR spin-trapping method (in Japanese). Food Style 3: 32-37, 1999.

16. Katayama M, Yoshimi N, Yamada Y, Sakata K, Kuno T, Yoshida K, Qiao Z, Vihn PQ, Iwasaki T, Kobayashi H and Mori H: Preventive effect of fermented brown rice and rice bran against colon carcinogenesis in male F344 rats. Oncol Rep 9: 817-822, 2002.

17. Kuno T, Hirose Y, Hata K, Kato K, Qiang SH, Kitaori N, Hara A, Iwasaki T, Yoshimura T, Wada K, Kobayashi $\mathrm{H}$ and Mori $\mathrm{H}$ : Preventive effect of fermented brown rice and rice bran on $\mathrm{N}$-nitrosomethylbenzylamine-induced esophageal tumorigenesis in rats. Int J Oncol 25: 1809-1815, 2004.

18. Katayama M, Sugie S, Yoshimi N, Yamada Y, Sakata K, Qiao Z, Iwasaki T, Kobayashi $\mathrm{H}$ and Mori $\mathrm{H}$ : Preventive effect of fermented brown rice and rice bran on diethylnitrosoamine and phenobarbital-induced hepatocarcinogenesis in male F344 rats. Oncol Rep 10: 875-880, 2003.

19. Togashi Y, Li Y, Kang J-H, Takeichi N, Fujioka Y, Nagashima K and Kobayashi H: D-penicillamine prevents the development of hepatitis in Long-Evans Cinnamon rats with abnormal copper metabolism. Hepatology 15: 82-87, 1992.

20. Kang J-H, Togashi Y, Kasai H, Hosokawa M and Takeichi N: Prevention of spontaneous hepatocellular carcinoma in LongEvan Cinnamon rats with hereditary hepatitis by the administration of D-penicillamine. Hepatology 18: 614-620, 1993.

21. Shibata t, Nagayasu H, Kawano T, Kitajo H, Hamada J-H, Moriuchi T, Okada F, Wananabe S, Yasuda S, Okuyama H, Kobayashi $\mathrm{H}$ and Arisue $\mathrm{M}$ : Unsaturated fatty acid feeding prevents the development of acute hepatitis in Long-Evans Cinnamon (LEC) rats. Anticancer Res 19: 5169-5174, 1999.
22. Samuni A, Chevion M and Czapski G: Unusual copper-induced sensitization of the biological damage due to superoxide radicals. J Biol Chem 256: 12632-12635, 1981.

23. Halliwell B: Oxidants and human disease: some new concepts. FASEB J 1: 358-364, 1987.

24. Esaki H, Kawakishi S, Morimitsu Y and Osawa T: New potent antioxidative o-dihydroxisoflavones in fermented Japanese soybean products. Biosci Biotechnol Biochem 63: 1637-1639, 1999.

25. Hirota A, Taki S, Kawai S, Yano M and Abe N: 1, 1-Diphenyl2-picrylhydrazyl radical scavenging compounds from soybean miso and antiproliferative activity of isoflavones from soybean miso toward the cancer cell lines. Biosci Biotechnol Biochem 64: 1038-1040, 2000.

26. Wu AH, Yang D and Pike MC: A meta-analysis of soyfoods and risk of stomach cancer; the problem of potential confounders. Cancer Epidemiol Biomarkers Prev 9: 1051-1058, 2000.

27. Ohara M, Lu H, Shiraki K, Ishimaru Y, Uesaka T, Katoh O and Watanabe H: Prevention by long-term fermented miso of induction of clonic aberrant crypt foci by azoxymethane in F344 rats. Oncol Rep 9: 69-73, 2002

28. Gitoh T, Yamada K, Ito A, Yin H, Kataoka T and Dohi K: Chemoprevention of N-nitroso-N-methylurea-induced rat mammary cancer by miso and tamoxifen, alone and in combination. Jpn J Cancer Res 89: 487-495, 1998.

29. Lanfear J, Fleming J, Walker M and Harrison P: Different patterns of regulation of the genes encoding the closely related $56 \mathrm{kDa}$ selenium and acetaminophen-binding proteins in normal tissues and during carcinogenesis. Carcinogenesis 14: 335-340, 1993.

30. Aquino TM, Porta EA, Sablan HM and Dorado RD: Effects of selenium supplementation on hepatocarcinogenesis in rats. Nutr Cancer 7: 25-36, 1985.

31. Le Boeuf RA, Laishes BA and Hoekstra WG: Effects of dietary selenium concentration on the development of enzyme-altered liver foci and hepatocellular carcinoma induced by diethylnitrosamine or N-acethylaminofluorene in rats. Cancer Res 45: 5489-5495, 1985. 